\title{
SEXUAEIDADE E DIFERENÇAS DE GÊNERO ENTRE JOVENS UNIVERSITÁRIOS
}

\author{
SEXUALITY AND GENDER DIFFERENCES AMONG \\ UNIVERSITY YOUTHS
}

Mauro L. Vieira ${ }^{1}$

Ana L. C. ${ }^{1}$

Silva Celiane $G$ Barghezan ${ }^{1}$

Daniela Mendes ${ }^{2}$

Crace Andreani ${ }^{2}$

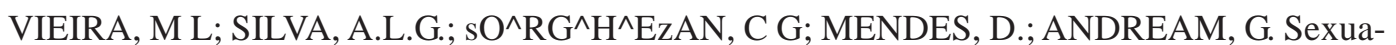
lidade e diferenças de género entre jovens universitários. Rev Bras. Cresc. Desenv Dum., São Paulo, 12(1),2002.

Resuma: O objetivo da presente pesquisa foi investigar aspectos relacionados com a ini-ciação sexual entre jovens. Foi aplicado um questionário a 42 homens e 36 mulheres com idade média de 22 anos. Os principais resultados foram: a) a iniciação sexual, em média, ocorreu mais cedo para os homens do que para as mulheres; b) apenas 14,3\% da amostra feminina avaliou positivamente a experiência em relação ao orgasmo durante o pnmeiro ato sexual, enquanto no caso dos homens esse valor foi consideravelmente maior (em torno de 85\%); c) a maioria das mulheres (63\%) relatou ter apenas um parceiro durante a vida sexual; entre os homens, $85 \%$ afirmou ter envolvimento sexual com duas ou mais parceiras até hoje. Conclui-se que entre os jovens pesquisados parecem existir acentuadas diferenças de género desde a percepção que esses jovens tem a respeito do papel que os pais tiveram durante a infancia, até características da iniciação sexual e outras aspectos relacionados à sexualidade.

Palavras-chaves: iniciação sexual; sexualidade; gênero; jovens; educação familiar

\section{INTRODUÇÃO}

A sexualidade na adolescência e entre jovens adultos é um assunto que preocupa profissionais de saúde, pois os riscos envolvidos na gravidez precoce ou indesejada e na transmissão de doenças sexualmente transin^jssíveis, tais como a AIDS, são consideráveis. Como não se conhece em detalhes as cal actetística3 da sexualidade entre os jovens, muitos problemas ainda existem em relação a uma efetiva educação sexual, incluindo programas institucionais ou orientações familiares, em países industrializados como os Estados Unidos, Canadá ou Suécia (RWSUVAARA, 1997) ou países em desenvolvimento como o Brasil (DIAS \& GOMES, 2000) e Peru (CHIRINOS e col., 2000). Entre os problemas identificados, pesquisas revelam que nem sempre o jovem recebe o apoio necessário e adequado para esclarecer dúvidas que surgem nesse período da vida (DIAS \& GOMES, 2000).

Especificamente entre adolescentes, "a iniciação sexual na adolescência na maioria dos ca-

1 Departamento de Psicologia, Universidade Pederal de Santa Catarina, Campus Universitário - Tnndade - Cep 88049-900 Florianópolis - SC - Brasil. Telefone: 48 331-9639 - Fax: + 5548 331-9639.

Correspondência para: E-mail: mvieira@uol.com.br. Professor do Departamento de Psicologia.

2 Acadêmicas do curso de graduação em Psicologia. 
sos, é uma manifestação de curiosidade natural diante do desenvolvimento físico e de maturação sexual, assim como pode ser expressão de amor e confiança. Pode também, estar relacionado com solidão, carência afetiva ou oportunidade de autoafirmação diante dos amigos, entre outros aspectos”. (COSTA e col., 1995, p. 152). Além disso, os autores também afirmam que, na adolescência, esses jovens não são suficientemente capazes para abstrair as conseqüências do comportamento sexual, deparando-se freqüentemente com situações de risco.

A sexualidade entre os jovens abarca um contexto muito amplo, como escolarização, estrutura familiar e estabilidade sócio-econômica da família, entre outros aspectos. De acordo com MEACHAM (2000), o emprego juvenil e a educação são elementos vinculados à sexualidade e ao comportamento reprodutivo. Com relação a isso, pode-se afirmar que uma menor educação e um maior desemprego são considerados fatores que favorecem uma maior atividade sexual nos jovens, juntamente com outros elementos como a urbanização acelerada, o adiamento do casamento e uma maturação sexual precoce. Especificamente no caso da menarca, evento que decorre da interação entre fatores genéticos, neuroendócrinos, psicológicos e ambientais, pode-se reafirmar que tem se apresentado cada vez mais cedo, segundo indicadores de diversos países (COSTA e col., 1995).

A diferença de gênero é um dos aspectos relevantes em relação à sexualidade. É importante considerar que os atributos dados às diferenças de gênero fazem parte de um amplo sistema envolvendo as dimensões biológica, psicológica, social, histórica e cultural. Diferenças de gênero podem ser explicadas a partir de uma perspectiva evolucionária, segundo a qual homens e mulheres seriam diferentes em função de características biológicas e psicológicas que foram selecionadas ao longo da evolução (FISHER, 1995; VIEIRA, 2000). Além disso, estudos tem mostrado que existe relação entre o nível de testosterona e a atividade sexual, como por exemplo, no início da atividade sexual, embora fatores psicológicos e sociais também exerçam papel importante (HALPERN e col., 1993).

Por outro lado, características peculiares de cada um dos sexos também são influenciadas por pressões sócio-culturais e históncas. Homens e mulheres mudaram bastante ao longo da história. No entanto, muitos esteriótipos permanecem influenciando as opiniões e atitudes das pessoas em relação ao sexo oposto. Segundo GOLDENBERG (1999), muitos homens tiveram poucas parceiras sexuais, fato que contraria os este- reótipos masculinos. Entretanto, a mulher continua sendo vista como frágil, indefesa e dependente dos homens.

GOLDENBERG (1988), falando sobre as diferenças na sexualidade de homens e mulheres, traz o depoimento de uma das mulheres que participou de sua pesquisa. Nele está bastante nítida a questão da sexualidade da mulher voltada para a satisfação do homem, sem que seja dado a ela o direito de sentir prazer. No mesmo depoimento, fica claro ainda que a idéia de repressão e submissão ao prazer para o homem, dá-se também pelo desconhecimento, por parte da mulher, do seu próprio corpo. Outro fator que auxilia na repressão da sexualidade feminina são os preceitos religiosos que se referem somente a um aspecto da sexualidade: o que visa a reprodução.

No caso dos jovens, as concepções sociais sobre o gênero têm grande influência na incorporação de modelos de masculinidade e feminilidade (ROUCO, 1999). É nesse contexto que as relações entre homens e mulheres adquirem significado, tanto nas interações sexuais como nas idéias de cada indivíduo como homem ou mulher e em sua identidade sexual.

Diferentes motivos levariam os jovens a terem as primeiras relações sexuais. O desejo sexual, a atração física, ou ainda o "ficar” - relação eventual, sem compromisso postenor, mesmo que não ocorra relação sexual - são alguns dos motivos declarados pelos rapazes (REITH, 1998). Para o homem, fica evidente a valorização do prazer genital centrado na penetração, no orgasmo e na virilidade. Para a maioria das garotas, são os compromissos afetivos envolvendo sentimentos de amor e ternura, e desejo de relacionamentos duradouros que provocam a relação sexual.

Além disso, existem muitos problemas enfrentados pelos jovens quando a sexualidade aflora. Nem sempre o jovem consegue acompanhar e compreender as transformações biológicas que acontecem com seu corpo, principalmente em relação à sexualidade. Um dos problemas mais graves que se tem na adolescência é a falta de informação, embora este não seja o único fator que explique os problemas decorrentes da sexualidade. No caso da mulher, a obtenção de prazer é limitada pelas exigências do parceiro e pelo desconhecimento do próprio corpo, embora dados atuais, ainda que pouco representativos, têm indicado que a mulher adolescente a cada dia tem conquistado liberdade e iniciativa em relação à sua sexualidade (DIAS \& GOMES, 2000).

Uma das tarefas que se coloca para a sociedade é identificar como o adolescente adquire informações sobre sexo. Através de uma análise histórica, pode-se constatar que as famílias for- 
necem informações diferenciadas a respeito da sexualidade para meninos e meninas. Meninos são mais instruídos sobre DST, Aids e relações sexuais, enquanto meninas são informadas sobre gravidez, virgindade menstruação (COSTA, 1986). Em geral, moças, mais do que rapazes, discutem assuntos relacionados a sexo com seus pais (LEBORD \& BARTH, 1992). Contudo, a rede de apoio, constituída por familiares, nem sempre está preparada para esclarecer dúvidas dos adolescentes em relação à sexualidade ou até mesmo aceitar a sexualidade dos adolescentes (DIAS \& GOMES, 2000).

Uma das repercussões da educação sexual recebida pelo jovem pode ser constatada no que se refere aos comportamentos de homens e mulheres. Os comportamentos masculinos levam à exibição de sua masculinidade, modo pelo qual se afirmam perante o seu grupo de relações. Essa prática é bastante diferenciada nas mulheres que tratam da sexualidade no âmbito privado, o que confere à sexualidade feminina idéias de segredos, zelos e valores a serem preservados.

Outros dados sobre a sexualidade entre jovens têm indicado que, na busca da identidade sexual, o adolescente oscila entre atividades masturbatórias e explorações genitais, podendo não haver, de fato, relações com penetração (TAVARES, 1999). No entanto, a primeira relação sexual vem se tornando cada vez mais precoce. Em Lima, Peru, foi encontrado que a idade da primeira relação sexual de adolescentes do sexo masculino é de 13 anos (CHIRINOS e col., 2000) e, no Brasil é de 13-14 anos (COSTA, 1986). Alguns autores têm procurado criar modelos para predizer a época, em termos de idade, da primeira relação sexual de rapazes. Comportamento delinqüente ou anti-social, envolvimento com drogas ilícitas ou legais e estrutura familiar não estável podem estar associados ao início precoce da atividade sexual interpessoal (CAPALDI e col., 1996). Por outro lado, esses autores afirmam que fatores psicológicas, tais como a ansiedade, pode atrasar a idade em que o intercurso sexual ocorre.

A ocupação dos adolescentes também parece ser um fator que interfere na atividade sexual. Pesquisadores do México (HUERTAFRANCO e col., 1996) têm encontrado que adolescentes, tanto moças como rapazes que trabalham, relataram significativamente maiores taxas de atividade sexual do que adolescentes estudantes. Na mesma pesquisa, também foi encontrado que entre rapazes sexualmente ativos, ser estudante ou trabalhador não afetou o uso de métodos contraceptivos. No entanto, a escolaridade influenciou no comportamento das moças: $27,5 \%$ das moças que estudavam informaram es- tar usando algum método contraceptivo, enquanto esse valor foi de apenas $9,5 \%$ para moças que trabalhavam.

Diante do exposto, pode-se ver que os jovens estão se tornando sexualmente ativos cada vez mais cedo e fora do casamento, de modo crescente (MEACHAM, 2000). No entanto, faltam dados consistentes sobre as várias dimensões da sexualidade nas diferentes fases evolutivas da vida. Dessa forma, um dos desafios que se coloca para os profissionais da saúde é responder questões no que se refere à saúde sexual e reprodutiva desde a adolescência, com o objetivo de criar programas de educação sexual efetivos.

A presente pesquisa teve como objetivo fazer um levantamento com jovens universitários sobre como eles classificariam pais e mães em relação à educação recebida, características da iniciação sexual, incluindo dados mais objetivos como a idade da primeira relação sexual e os sentimentos envolvidos nessa experiência, além de obter algumas informações sobre a vida sexual atual.

\section{MÉTODO}

\section{Sujeitos}

Participaram da presente pesquisa 78 jovens universitários freqüentando cursos de diferentes áreas do conhecimento. Do total, 42 eram do sexo feminino (53,8\% da amostra) e 36 eram do sexo masculino (46,2\% da amostra). A média de idade dos homens foi de 21,84 anos e das mulheres 22,4 anos. A ampla maioria da amostra era composta de pessoas solteiras: 77\% das mulheres e 91,7\% dos homens. A religião de criação dos sujeitos de ambos os sexos era predominantemente católica (69,05\% das mulheres e 88,88\% dos homens).

\section{Instrumento da pesquisa e procedimentos}

Para a realização da pesquisa foi utilizado um questionário elaborado por Nelson Vitiello, obtido pela rede mundial de computadores (www.sbrash.org.br/) em que estava disponível para realização de pesquisa. Antes da aplicação, o questionário foi revisado e algumas adaptações foram realizadas.

O questionário consistiu de quatro partes distintas: a) dados gerais dos indivíduos; b) educação recebida na infância; c) informações sobre iniciação sexual; e d) dados sobre a vida sexual atual. O instrumento apresentava um total de 37 questões. Em cada questão a pessoa deveria escolher uma dentre várias alternativas possíveis.

A aplicação do instrumento foi realizada de forma coletiva (salas de aulas). Antes de responder ao questionário, os participantes foram 
informados que a participação era voluntária e não havia necessidade de identificação pessoal. Foi mencionado que era necessário uma leitura atenta e o preenchimento deveria ser completo e feito de maneira sincera. Foram colocados à disposição telefone e e-mail para esclarecimentos de quaisquer dúvidas sobre a pesquisa e obtenção de informação sobre os resultados da mesma.

\section{RESULTADOS}

A análise dos resultados foi realizada com base em três itens: a) educação familiar recebida; b) características da primeira relação sexual; e c) vida sexual atual.

\section{PAPEL DO PAI E DA MÃE NA EDUCAÇÃO DE FILHOS E FILHAS}

Com relação às mulheres, os pais foram classificados como mais omissos e menos educadores do que a mãe (Figura 1). A maioria das filhas classificou a mãe como educadora $(70,1 \%)$ e apenas 17,4\% como omissa.

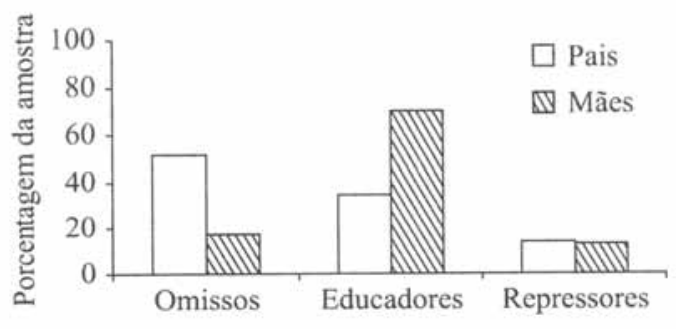

Figura 1: Classificação de pais e mães, pelas filhas, em relação à educação sexual recebida na infância.

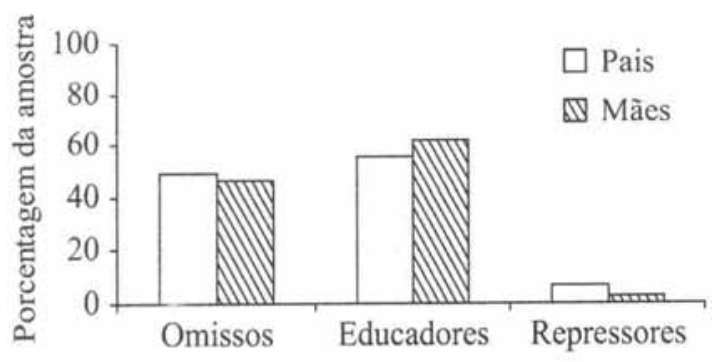

Figura 2: Classificação de pais e mães, pelos filhos (homens), em relação à educação sexual recebida na infância.

Contudo, entre os homens, pais e mães foram classificados praticamente da mesma forma em relação ao estilo de educação, embora as mães tenham sido caracterizadas como mais educado- ras e um pouco menos omissas do que os pais (Figura 2). O papel de repressor, tanto por parte do pai como da mãe, indicou ser inaior no caso das filhas. Os dados, no conjunto, indicam diferenças de gênero no estilo de educação dos pais em relação aos filhos.

\section{PRIMEIRA RELAÇÃO SEXUAL}

Entre os homens, 79\% tiveram a primeira relação sexual entre 15 e 18 anos. No caso das mulheres, 80\% destas tiveram a iniciação sexual no período entre 16 e 20 anos. Constate-se também, na figura 3, que $9 \%$ dos homens tiveram a iniciação sexual com 13 anos e apenas 6\% com 19 anos. Entre as mulheres, cerca de 23\% tiveram o primeiro relacionamento sexual entre $20 \mathrm{e}$ 22 anos (Figura 3).

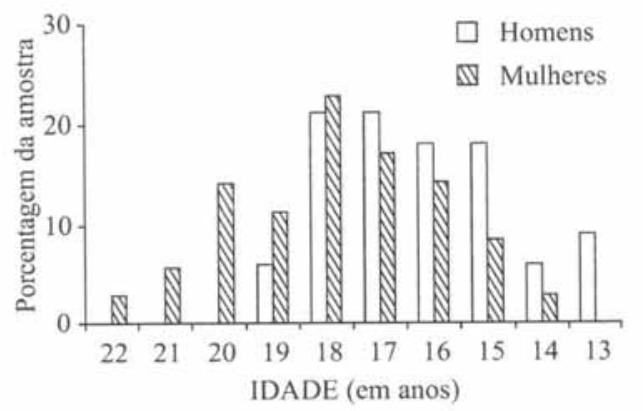

Figura 3: idade (em anos) da primeira relação sexual relatada por rapazes e mulheres.

Constatou-se que, entre as mulheres, $80 \%$ afirmaram que o namorado foi o parceiro da iniciação sexual. No caso dos homens, a familiaridade também foi importante na escolha do parceiro durante a primeira relação sexual. Contudo, nem sempre envolvia um parceiro com envolvimento afetivo-sexual mais duradouro. A primeira relação sexual dos homens, em apenas 35\% dos casos, foi com a namorada. Em outros 35\% da amostra, a parceira era apenas unta conhecida (Figura 4).

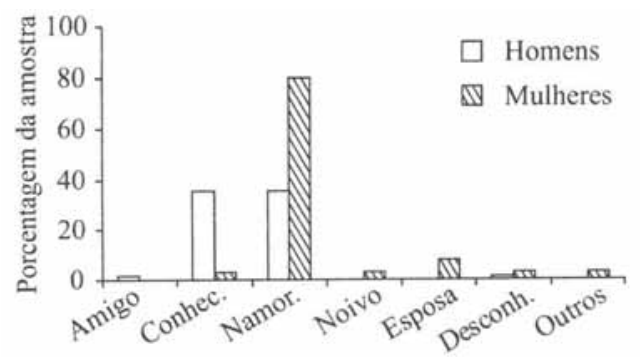

Figura 4: Tipos de relacionamentos que homens e mulheres tinham com o parceiro quando ocorreu a primeira relação sexual. 
Com relação ao grau de insatisfação na primeira relação sexual, também constatou-se diferenças dependendo do sexo dos indivíduos. Em todos os itens (desejo, excitação e orgasmo) apareceu algum grau de insatisfação na amostra feminina, embora em relação ao orgasmo, o número de mulheres que relatou ter algum grau de insatisfação chegou a 85,7\% da amostra. No caso dos homens, além da insatisfação ser acentuadamente menor em dois itens, foi inexistente com relação ao desejo (Figura 5).

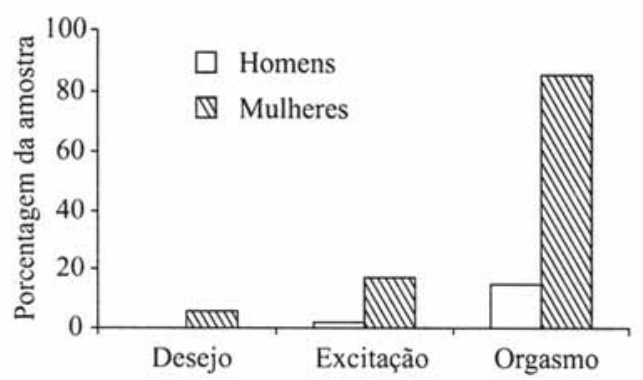

Figura 5: Insatisfação relatada por homens e mulheres durante a primeira relação sexual a respeito do desejo, excitação e orgasmo.

\section{NÚMERO DE PARCEIROS}

A maioria das mulheres (63\%) relatou ter apenas um parceiro durante a vida sexual, enquanto que cerca de $25 \%$ da amostra af $\sim$ rmou ter de 2 a 5 parceiros. Entre os homens, 85\% da amostra relatou ter dois ou mais parceiros, não havendo praticamente diferença no número de indivíduos que responderam ter de 2 a 5 parceiras ou 10 ou mais. Em ambos os casos a porcentagem foi em torno de $26,5 \%$ (Figura 6 ).

Por fim, o resultado com relação à crença de ter algum problema sexual foi bastante próximo entre os jovens universitários, i dependentemente do sexo. A ampla maioria das pessoas que participaram da pesquisa disse não ser portadora de problema sexual (Figura 7).

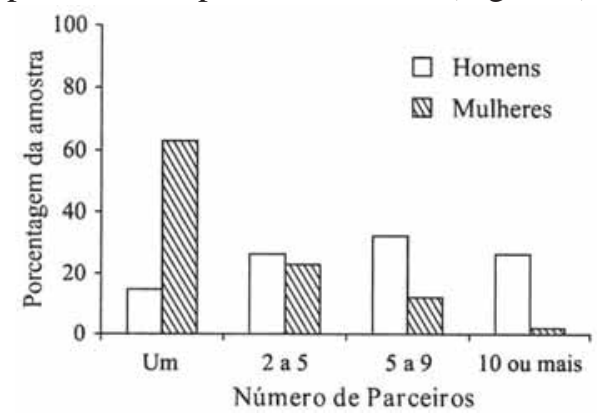

Figura 6: Número de parceiros que homens e mulheres tiveram durante a vida sexual

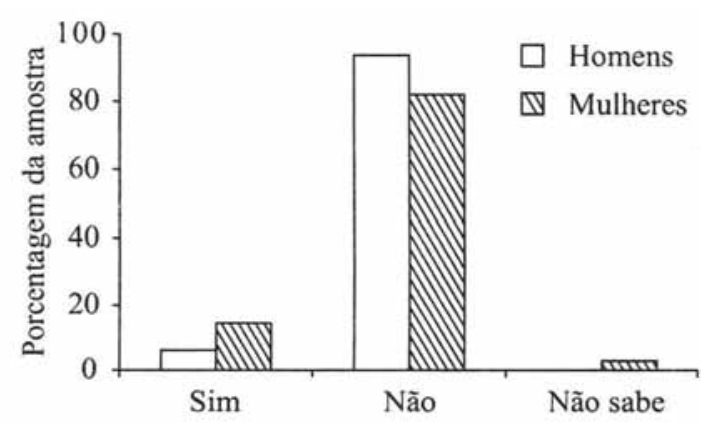

Figura 7: Percepção de homens e mulheres sobre serem ou não portadores de algum problema sexual.

\section{DISCUSSÃO}

Os dados da presente pesquisa indicaram algumas semelhanças e acentuadas diferenças de gênero na educação recolhida, iniciação sexual e vida sexual de jovens universitários.

\section{PAIS/MÃES E FILHOS/FILHAS}

Os jovens avaliaram de forma diferente 0 papel que o pai e a mãe tiveram durante a infância. Por exemplo, no caso dos homens, não houve grande diferença no papel do pai ou da mãe em relação à educação recebida, embora a mãe tenha sido classificada mais como educadora do que omissa pelos filhos. Isso parece indicar a existência de estereótipos de diferenças de gênero dos pais em relação aos filhos e filhas. Mesmo com as transformações que a sociedade vem sofrendo ao longo dos anos, ainda cabe mais à mãe do que ao pai a responsabilidade da educação dos filhos e filhas, incluíndo a educação sexual.

A partir desse dado, uma das questões que pode surgir é a seguinte: que tipo de educação os filhos e filhas recebem em casa e quais os efeitos sobre o comportamento, de um modo geral, e sobre o comportamento sexual de modo específico? Outra questão é: quais as fontes que os jovens têm para adquirir informações e apoio diante da sexualidade? Um estudo mostrou que o interlocutor preferencial de adolescentes do sexo feminino de classe média-baixa para obter informação sobre prevenção e gravidez na adolescência é a mãe (DIAS \& GOMES, 2000). No entanto, nesse mesmo estudo, foi encontrado que as adolescentes gestantes relataram que as informações que elas obtiveram das mães foram percebidas como parciais e incompletas, além de haver falta de confiança na mãe. Além disso, a rede de apoio (tias e amigas, por exemplo) nem sempre 
foi eficaz. Outro estudo, realizado nos Estados Unidos, também indicou que as mães não estavam adequadamente preparadas para responder questões relacionadas à sexualidade de seus filhos (HOCKEMBERRY ecol., 1996).

Pesquisas têm procurado identificar aspectos da educação sexual que adolescentes recebem de familiares e amigos. Por exemplo, KELLEY e col. (1999) fizeram um levantamento sobre conteúdo, característica e grau de ‘conforto’ de conversas sobre sexualidade entre adolescentes estadunidenses de origem africana, com idades entre 13 e 15 anos, e seus pais. Os resultados da pesquisa mostraram que tanto moças como rapazes foram mais propensos para conversar assuntos relacionados à sexualidade com as mães do que com os pais, o que confirma os dados obtidos na presente pesquisa. Por outro lado, rapazes tenderam a conversar mais sobre sexo com seus pais do que as moças. Esses dados também são confirmados aqui no Brasil na presente pesquisa. Contudo, a fonte mais freqüente que os jovens estadunidenses procuravam para conversar sobre sexo eram os amigos.

Embora mãe e pai possam não ser a fonte principal de consulta sobre sexualidade, eles têm grande importância no comportamento sexual dos jovens estadunidenses (op. cit.). Quando os adolescentes de ambos os sexos conversavam mais sobre sexo com suas mães do que com amigos, ele ou ela era menos propenso a ter relações sexuais precoce e mais provável ter valores conservadores. Em outro estudo, mostrou-se que a desaprovação por parte de pais e mães da experiência sexual precoce foi associada ao retardo no início da relação sexual (RESNICK e col., 1997).

Além da educação sexual ou da atitude de pais e mães em relação à sexualidade na adolescência, a própria situação dos pais também pode influenciar o comportamento sexual dos adolescentes. Estrutura familiar não estável foi um dos fatores associados com o início precoce da atividade sexual (CAPALDI e col., 1996).

\section{INICIAÇÃO E VIDA SEXUAL}

A iniciação sexual pode ter vários significados. Por exemplo, o primeiro ato sexual pode ser entendido como um rito de passagem para a vida a adulta. Se isto for considerado, constata-se que o início da maturidade está sendo antecipado em relação a algumas décadas atrás. Em diversos países, constatou-se que a idade em que o jovem tem o início da atividade sexual é bastante precoce. Em algumas cidades como Lima (Peru), estudo feito em uma escola de ensino médio, indicou que muitos adolescentes iniciaram a atividade sexual com 13 anos (CHIRINOS e col., 2000). No Brasil, igualmente, a iniciação sexual para muitos jovens ocorre por volta dos 13-14 anos (COSTA, 1986).

Embora se saiba que o desenvolvimento físico, desde o nascimento, é geralmente mais rápido em pessoas do sexo feminino, constatou-se na presente pesquisa que as mulheres iniciaram um pouco mais tarde o intercurso sexual e o grau de satisfação com essa experiência foi menor do que o dos homens, principalmente em relação ao orgasmo. Conclui-se, portanto, que também, em relação à iniciação sexual, parece existir diferenças de gênero.

Na presente pesquisa, encontrou-se que a iniciação sexual dos jovens universitarios pesquisados ocorreu em épocas um pouco mais tarde do que é registrado na literatura. Os homens tiveram a primeira relação sexual por volta dos 16 anos e as mulheres em torno de 18 anos. Pesquisas têm demonstrado que existem alguns fatores que podem explicar o período em que a primeira relação sexual ocorre. O início precoce pode estar associado com a desestrutura familiar, comportamento anti-social ou envolvimento com drogas - lícitas ou ilícitas (CAPALDI e col., 1996). Além disso, a ocupação do adolescente também parece ser um fator que tem conseqüências sobre a atividade sexual do mesmo. Foi encontrado em uma pesquisa realizada nos Estados Unidos que a prática de sexo era mais freqüente entre adolescentes que só trabalhavam em comparação com adolescentes que só estudavam (HUERTA FRANCO e col., 1996). Na presente pesquisa, o grau de satisfação com a experiência sexual inicial foi menor nas mulheres. Estas também mencionaram ter menor número de parceiros e a iniciação sexual envolver principalmente o namorado o que demonstra que a iniciação sexual das mulheres, de um modo geral, está altamente relacionada à afetividade e à intimidade.

Parece que os homens procuram relações sexuais para obter prazer o que pode ser alcançado facilmente com diversos parceiros, enquanto as mulheres são movidas para as relações sexuais quando estão envolvidas emocionalmente, o que limita o número de parceiros. Diversas pesquisas analisadas a partir da perspectiva evolucionária têm explicado diferenças de gênero nessa direção. O homem teria maior predisposição para dissociar sexo de envolvimento afetivo do que a mulher (FISHER 1995; VIEIRA, 2000; OTTA \& QUEIROZ, 1998; KENRICK e col., 1989).

Com relação aos sentimentos envolvidos na primeira relação sexual, as explicações parecem ser mais complexas. A mulher, apesar de todo 
o seu potencial para experienciar o orgasmo, parece apresentar dificuldade maior nessa área do que os homens durante a iniciação sexual. Talvez o medo da gravidez e a cobrança mais acentuada por parte dos pais ou da própria sociedade do que em relação aos rapazes, associada com a falta de conhecimento sobre sua sexualidade, são fatores que podem estar envolvidos na explicação desse fenômeno. Além disso, a educação sexual recebida pode levar o homem a se exibir e a mulher a se reprimir. Para o homem, quanto mais cedo tiver a sua primeira relação sexual, maior será a prova de sua masculinidade perante seu grupo. Sua virilidade será provada para todos e a questão da homossexualidade se afastará. Essa iniciação pode, em muitos casos, vir a ser com uma mulher desconhecida, em prostíbulos, com mulheres mais velhas e "mais experientes". No caso na Iniciação sexual feminina, a situação e bem diferente. A primeira experiência sexual não será com qualquer homem. Primeiro ela precisa conhecê-lo para depois ter um relacionamento mais íntimo. Talvez seja por isso que, geralmente, a iniciação sexual feminina dá-se no namoro, o que não é fato tão comum no caso dos homens. Essa diferenciação de gênero ficou evidente nos resultados da presente pesquisa.

Diante do exposto, comparando dados obtidos na pesquisa com dados da literatura, constata-se a necessidade de um conhecimento mais aprofundado da sexualidade humana de um modo geral e, da sexualidade entre adolescentes e adultos jovens, de modo mais específico. Esse conhecimento certamente irá representar um marco decisivo na elaboração de programas mais efetivos de educação sexual, tanto no nível institucional como no familiar. Isso tem como objetivo fazer com que o adolescente viva a sua sexualidade com prazer e responsabilidade, procurando evitar problemas relacionados com a gravidez precoce e não desejada, além da transmissão de doenças sexualmente transmissíveis, incluíndo nesse a AIDS.

\begin{abstract}
The purpose of the present study was to examine aspects related to the initiation of sexual activity among young people. Forty-two men and thirty-six women ( $\mathrm{M}=22$ years old) answered a questionnaire. Main results showed that: a) the first sexual intercourse, in general, occurred earlier to men than to women; b) only $14.3 \%$ of women assessed positively the experience in relation to orgasm during the first sexual intercourse, while among men this value was considerably higher (about $85 \%$ ); c) the majority of women (63\%) reported to have had only one partner during their sexual life; $85 \%$ of men reported to have had sexual involvement with two or more women until that moment. In conclusion, it appears that among the young people who participated in the research, there are gender differences in the perception they have of the role that their parents had throughout their childhood, the characteristics of the initiation of their sexual activity and other aspects of sexuality.
\end{abstract}

Key-words: sexual initiation; sexuality; gender differences; young people; family education.

\section{REFERENCIAS BIBLIOGRÁFICAS}

CHIRINOS, J.L.; SALAZAR, V.C.; BRINDIS, C.D. A profile of sexually active male adolescente high school students in Lima, Peru. Cadernos de Saúde Pública, 16: 733-746, 2000.

CAPALDI, D.M.; CROSBY, L.; STOOLMILLER, M. Predicting the timing of first sexual intercourse for at risk adolescent males. Child Dev., 67: 344-349, 1996.

COSTA, M. Iniciação Sexual. In: COSTA, M. Sexualidade na adolescência: dilemas e crescimento. $3^{\mathrm{a}}$ ed. Porto Alegre: L \& PM, 1986. p.96-104.

COSTA, M.; PINHO, J.; MARTINS, S. Aspectos psicossociais e sexuais de gestantes adolescentes em Belém-Pará. Jornal de Pediatria, 17: 151-157, 1995.
DIAS, A. C. G.; GOMES, W.B. Conversas, em família, sobre sexualidade e gravidez na adolescência: percepcão das jovens gestantes. Psicologia, Reflexão e Crítica, 13: 109-125, 2000.

FISHER, H. Anatomia do amor. Rio de Janeiro: Eureka, 1995. (Tradução Magda Lopes e Maria Carbajal)

GOLDENBERG, M. A. A. Luta contra desigualdade sexual: "Dois pesos e duas medidas". In: GOLDENBERG, M. A. A. Educação sexual: uma proposta um desafio. São Paulo: Cortez, 1988. p.15-40.

GOLDENBERG, M. A. A. Homem/mulher: o que existe de novo? In: GOLDENBERG, M. A. A. O prazer e o pensar. São Paulo: Gente, 1999. p.155-160. 
MEACHAM, D. Sexualidad y salud reproductiva de adolescentes: el desafio del 2000. In: MEACHAM, D. Mujer Salud. México, 2000. p. 31-54.

OTTA, E.; QUEIROZ, R. A sexualidade humana numa perspectiva interdisciplinar. In: SOUZA, L. S. ; FREITAS, M. F. Q.; RODRIGUE, M. M. R (Org.). Psicologia. Reflexões (im)pertinentes. São Paulo: Casa do Psicólogo, 1998. p. 225-246.

RESNICK, M. D.; BEARMAN, P. S.; BLUM, R. W.; BAUMAN, K. E.; HARRIS, K. M.; JONES, J. T. J.; BEUHRING, T.; SIEVING, R. E.; SHOW, M.; IRELAND, M.; BEARING, L. H.; UDRY, JR. Protecting adolescents from harm. JAMA, 278: 823-832, 1997.
RIETH, F. Ficar e namorar. In: BRUSCHINI, C.; HOLLANDA, H. B. (Orgs.). Horizontes plurais: novos estudos de gênero no Brasil. São Paulo: ECC e Ed.34, 1998. p.l 11-133.

ROUCO, J. M. Adolescência ou adolescências? In: RIBEIRO, M. O prazer e o pensar: São Paulo: Gente,1999. p. 93-99.

RUUSUVAARA, L. Adolescent sexuality: an educational and counseling challenge. Ann. $N$. Y Acad. Set., 816: 411-413, 1997.

TAVARES, R. M. Aspectos psicológicos. In: RIBEIRO, M. O prazer e o pensar: São Paulo: Gente, 1999. p.87-92.

VIEIRA, M. L. Contribuições da etologia para a compreensão do comportamento humano. In: Anais, 50. Florianópolis: Encontro Anual de Etologia, 2000. p. 11-16.

Recebido em 04/08/2001

Modificado em 23/09/2001

Aprovado em 20/11/2001 